\title{
SATISFACCIÓN DE LOS ALUMNOS CON LA INSTITUCIÓN UNIVERSITARIA Y EL RENDIMIENTO ACADÉMICO
}

\section{SATISFACTION OF STUDENTS WITH UNIVERSITY INSTITUTION AND ACADEMIC PERFORMANCE}

Abarca Franco, S. Cáceres Galera, S., Jiménez Ortiz, E., Moraleda Borja, V. y Romero Durán, B. Universidad de Granada

\section{Resumen}

En el presente artículo, se ha investigado la relación existente entre el rendimiento académico y la satisfacción con la institución educativa en alumnos universitarios. Nuestra hipótesis de partida era que se hallaría una correlación positiva entre ambas variables. Para ello, se utilizó una muestra de 101 estudiantes de Licenciatura en Psicología de la Universidad de Granada. El instrumento de evaluación que se ha utilizado es el SEUE (Satisfacción de los Estudiantes Universitarios con su Educación) de Gento y Vivas (2003). Este instrumento incluye una serie de subdimensiones acerca del constructo estudiado que hemos tenido en cuenta en el análisis estadístico. Tras el análisis de los resultados hemos comprobado que no se ha confirmado la hipótesis, ya que no se observa una correlación significativa entre las variables estudiadas. Junto con la discusión de los resultados, incluimos una serie de limitaciones a tener en cuenta para futuras investigaciones.

Palabras Claves: Rendimiento académico, Satisfacción, Universidad.

\begin{abstract}
In the present article, we have investigated the relationship between academic performance and satisfaction with the educational institution in university students.

Our hypothesis was that we would be found a positive correlation between both variables. For this, we used a sample of 101 students of degree in psychology at the University of Granada. The assessment tool that has been used is the SEUE (Satisfacción de los Estudiantes Universitarios con su Educación) of Gento and Vivas (2003). This instrument includes some subdimensions about studied construct which we have taken into account in the statistical analysis. After the analysis of the results we have verified that not been confirmed the hypothesis, since there isn't a significant correlation between the variables studied. Along with the discussion of the results, we have included a series of limitations to keep in mind for future research.
\end{abstract}

Keywords: Academic performance, Satisfaction, University.

\section{Introducción}

La satisfacción es el resultado de un proceso que se da en el sujeto, por lo que se trata de un fenómeno esencialmente subjetivo. Es un concepto que se refiere a algo o alguien, que tiene que ver, con lo que el sujeto desea y con el resultado que espera. Para lograr la satisfacción, como algo sentido en un sujeto, debe haber al menos una intención por parte de otro sujeto o institución de realizar una acción determinada que provoque un resultado, el cual será valorado como positivo o no (Zas, 2002).

El aumento de la calidad de la institución académica, constituye actualmente uno de los principales retos a los que se enfrenta la Universidad española. En este sentido, en 
las últimas dos décadas las universidades de nuestro país han puesto en marcha metodologías de evaluación de la calidad orientadas a la mejora institucional. Para ello es importante realizar evaluaciones sobre la satisfacción de los estudiantes con el centro como propuso Pereira (2011).

La satisfacción ha sido estudiada en el ámbito de las instituciones educativas para ver cómo se relaciona con diferentes variables relativas a los estudiantes. Algunas de estas variables han sido burnout, absentismo escolar, compromiso, autoeficacia, rendimiento académico, etc. (Caballero, Abello y Palacio, 2007). Otros estudios se han centrado en la relación existente entre los resultados académicos y la satisfacción de los estudiantes con la enseñanza recibida en todo su proceso, es decir, hasta la comprobación de conocimientos (Fernández, Fernández, Álvarez y Martínez, 2007). Así mismo, en el estudio de Cabrera y Galán (2002) uno de los objetivos principales era probar que las expectativas positivas que posee el alumno sobre la universidad, se asocian a un alto rendimiento académico. Según el estudio de Jiménez, Terriquez y Robles (2011), la satisfacción del estudiante refleja la eficiencia de los servicios académicos y administrativos. Por ello es importante conocer la satisfacción de los estudiantes respecto a las unidades de aprendizaje, las interacciones con profesores y compañeros de clase, así como con las instalaciones y el equipamiento.

Los hallazgos de Caballero et al., (2007) muestran que el buen funcionamiento académico correlaciona positivamente con la satisfacción y viceversa. Sin embargo, otros estudios como el de Hon, Raslin y Thean (2011) encontraron que los alumnos más satisfechos eran los que obtenían calificaciones más bajas. De acuerdo con Garbanzo (2007), en el rendimiento académico del estudiante universitario intervienen diversos factores: personales, sociales e institucionales. Entre los factores institucionales se encuentran el ambiente estudiantil, las condiciones institucionales, los servicios institucionales de apoyo, las relaciones profesor-estudiante, etc.

Montero, Villalobos y Valverde (2007) han estudiado la relación entre factores institucionales, pedagógicos, psicosociales y sociodemográficos con el rendimiento académico, encontrando entre otros resultados, que hay una relación positiva entre la satisfacción del alumno con el docente y el rendimiento académico.

Se considera que es importante identificar los obstáculos y facilitadores que los estudiantes encuentran en el ámbito académico, y la relación de estos con el bienestar psicológico y desempeño académico (Soria, Martínez, Bresó, Llorens y Grau, 2005).

Con el fin de evaluar la satisfacción de los estudiantes con la institución escolar, Hussain y Bhamani (2012) han creado el instrumento de medida Student University Satisfaction Scale (SUSS). En su estudio, el instrumento demostró su fiabilidad y validez, obteniendo un coeficiente alfa de Cronbach de 0.91. Dado que este instrumento está desarrollado para población pakistaní, en este estudio utilizaremos el cuestionario Satisfacción de los Estudiantes Universitarios con su Educación (SEUE), de Gento y Vivas (2003), validado para la población española, y que presenta un coeficiente alfa de Cronbach de 0,93.

De acuerdo con los estudios revisados, consideramos importante profundizar en el estudio de la relación entre variables relativas a la actuación del estudiante y a la evaluación de este sobre el centro de estudios.

En el presente estudio se pretende investigar si existe relación entre la satisfacción de los alumnos con el centro universitario en el que estudian y su rendimiento académico. En concreto, nuestra hipótesis de investigación es la siguiente:

Existe una relación positiva entre la satisfacción con el centro y el rendimiento académico, de modo que a mayor satisfacción del estudiante con el centro, mayor será el rendimiento académico obtenido. 


\section{Método}

\section{Participantes}

La muestra está compuesta por 101 alumnos (25 hombres y 76 mujeres) de la Universidad de Granada, de la Facultad de Psicología, con edades comprendidas entre 19 y 30 años, la media de edad fue de 22.26 (DT=1.95).

\section{Materiales y procedimiento}

A los participantes se les entregó el cuadernillo en el cuál aparecían las instrucciones que debían seguir, y que además se explicaron oralmente por si tenían alguna duda.

El cuadernillo estaba formado por:

- Datos personales referentes a la edad, sexo y nota media del expediente académico.

- Un cuestionario de Gento y Vivas (2003), Satisfacción de los Estudiantes Universitarios con su Educación (SEUE). Este instrumento consta de las siguientes dimensiones: Condiciones básicas de infraestructura $(\mathrm{Cl})$, Servicios de la Institución (SI), Condiciones de seguridad (S), Consideración a su situación económica (CS), Seguridad emocional por el trato afectuoso (SE), Sentido de pertenencia a la Institución y al grupo de clase por la aceptación (PE), Proceso de enseñanza y aprendizaje (EA), Logros personales (LP), Reconocimiento del éxito personal (RE) y Autorrealización (AU). La consistencia interna de la escala calculada mediante el coeficiente alfa de Cronbach es de 0.93.

El cuadernillo se pasó a lo largo de un día, en tres grupos de clase, de la asignatura de Psicología de los Grupos y las Organizaciones de $4^{\circ}$ curso de Licenciatura en Psicología. También se pasó dicho cuadernillo a alumnos de otros cursos que se encontraban en la biblioteca de la facultad en ese día.

\section{Resultados}

Tras la recogida de datos se han llevado a cabo análisis correlacionales entre la nota media y la satisfacción (global y subdimensiones), además de estadísticos descriptivos (media y desviación típica). En las siguientes tablas se muestran los resultados obtenidos en dichos análisis.

Tabla 1. Descriptivos de las dimensiones de la satisfacción

\begin{tabular}{lcc}
\hline & $\mathrm{M}$ & $\mathrm{DT}$ \\
\hline Nota expediente & 7.43 & 0.76 \\
$\mathrm{CI}$ & 39.99 & 7.07 \\
$S I$ & 43.56 & 8.72 \\
$S$ & 18.85 & 4.86 \\
$C S$ & 18.07 & 5.10 \\
$S E$ & 25.83 & 5.03 \\
$P E$ & 31.66 & 6.57 \\
$E A$ & 51.46 & 13.13 \\
LP & 20.02 & 4.64 \\
$R E$ & 15.02 & 3.80 \\
$A U$ & 18.80 & 5.17 \\
$S T$ & 282.87 & 45.57 \\
\hline
\end{tabular}


Tabla 2. Correlación entre las dimensiones de la satisfacción y el rendimiento

\begin{tabular}{|c|c|c|c|c|c|c|c|c|c|c|c|}
\hline & $\mathrm{Cl}$ & $S I$ & $S$ & CS & $S E$ & $P E$ & $E A$ & $L P$ & $R E$ & $A U$ & $S T$ \\
\hline Nota & $.085^{*}$ & $-.092^{*}$ & $-.127^{*}$ & $-.07^{*}$ & $-.118^{*}$ & $-.066^{*}$ & .018 & $.063^{*}$ & .015 & $-.067^{*}$ & .047 \\
\hline Expediente & & & & & & & & & & & \\
\hline
\end{tabular}

Nota: ${ }^{*} \mathrm{p}<.05$; $\mathrm{Cl}$ : Condiciones básicas de Infraestructura; SI: Servicio de la Institución; S: Condiciones de Seguridad; CS: Consideración de la Situación Económica; SE: Seguridad Emocional; PE: Sentido de Pertenencia; EA: Proceso de Enseñanza aprendizaje; LP: Logro Personal; RE: Reconocimiento del Éxito Personal; AU: Autorrealización; ST: Satisfacción Total.

No se obtiene una correlación significativa entre la Satisfacción Total (ST) y la nota media del expediente académico de los universitarios.

Sin embargo, la nota media del expediente académico correlaciona positivamente con las dimensiones: Condiciones básicas de Infraestructura (Cl) y Logro Personal (LP). Por otro lado, esta correlaciona negativamente con las dimensiones: Servicio de la Institución (SI), Condiciones de Seguridad (S), Consideración de la Situación Económica (CS), Seguridad Emocional (SE), Sentido de Pertenencia (PE), Autorrealización (AU).

Tabla 3. Correlaciones entre las subdimensiones de la escala

\begin{tabular}{|c|c|c|c|c|c|c|c|c|c|}
\hline & SI & $S$ & CS & SE & $P$ & EA & LP & RE & $A U$ \\
\hline $\mathrm{Cl}$ & $.508^{\star \star}$ & $.544^{\star \star}$ & $.415^{\star \star}$ & $.400^{\star \star}$ & $.369^{* *}$ & $.475^{\star \star}$ & $.288^{\star \star}$ & .191 & $.344^{* *}$ \\
\hline SI & & $.560^{* *}$ & $.504^{* *}$ & $.428^{* *}$ & $.402^{* *}$ & $.366^{* *}$ & $.314^{\star \star}$ & $.277^{* *}$ & $.375^{* *}$ \\
\hline$S$ & & & $.523^{* *}$ & $.538^{\star *}$ & $.453^{\star *}$ & $.401^{* *}$ & $.394^{\star \star}$ & $.263^{* \star}$ & $.338^{* *}$ \\
\hline CS & & & & $.489^{\star *}$ & $.490^{* *}$ & $.345^{* *}$ & 177 & $.276^{* *}$ & $.297^{* *}$ \\
\hline SE & & & & & $.699^{* *}$ & $.612^{* *}$ & $.347^{\star \star}$ & $.469^{\star *}$ & $.339^{* *}$ \\
\hline$P$ & & & & & & $.639^{* *}$ & $.379^{\star \star}$ & $.549^{\star *}$ & $.450^{* *}$ \\
\hline EA & & & & & & & $.525^{\star \star}$ & $.520^{\star \star}$ & $.628^{* *}$ \\
\hline LP & & & & & & & & $.475^{\star \star}$ & $.643^{* *}$ \\
\hline RE & & & & & & & & & $.407^{* *}$ \\
\hline
\end{tabular}

Nota: ${ }^{* *}$ p<.001; Cl: Condiciones básicas de Infraestructura; SI: Servicio de la Institución; S: Condiciones de Seguridad; CS: Consideración de la Situación Económica; SE: Seguridad Emocional; PE: Sentido de Pertenencia; EA: Proceso de Enseñanza aprendizaje; LP: Logro Personal; RE: Reconocimiento del Éxito Personal; AU: Autorrealización; ST: Satisfacción Total.

Como se puede observar en la Tabla 3, las diferentes su dimensiones que componen la escala SEUE correlacionan de manera positiva entre sí, siendo las correlaciones estadísticamente significativas.

\section{Discusión}

Tras la revisión de la literatura acerca de la satisfacción de los universitarios con el centro de estudios, hemos podido comprobar que esta variable se ha relacionado con diversos factores. Por ejemplo Caballero et al., (2007), encontraron que se relacionaba 
con el burnout, absentismo escolar, compromiso, autoeficacia, rendimiento académico, etc.

De todas las variables implicadas, nos hemos centrado en la revisión de estudios que relacionan la satisfacción con el rendimiento académico. Algunos autores que han estudiado esta temática han sido Cabrera y Galán (2002) y Caballero et al. (2007) cuyos hallazgos muestran que el buen funcionamiento académico correlaciona positivamente con la satisfacción y viceversa.

Partiendo de esta base, nuestro objetivo ha sido investigar la relación entre la satisfacción de los alumnos con el centro universitario en el que estudian y su rendimiento académico. Nuestra hipótesis de partida era que se daría una correlación positiva entre ambas.

Sin embargo, a la luz de los hallazgos obtenidos, debemos rechazar la hipótesis ya que no hemos encontrado una correlación estadísticamente significativa. Por tanto, nuestros resultados no coinciden con las investigaciones citadas.

Por otra parte, para profundizar más en el constructo de satisfacción con el centro, hemos tenido en cuenta diferentes subdimensiones que recoge el instrumento SEUE de Gento y Vivas (2002). Una de estas subdimensiones es la de satisfacción con el proceso de enseñanza-aprendizaje, la cual fue estudiada por Fernández et al. (2007), obteniendo una correlación positiva con el rendimiento. En cambio, la correlación obtenida para esta subdimensión en nuestro estudio no es significativa.

Aunque la mayoría de datos obtenidos en la literatura aportan correlaciones positivas entre ambas variables, algunos estudios muestran resultados contrarios. Un ejemplo sería la investigación de Hon et al. (2011), en la cual encontraron que los alumnos más satisfechos eran los que obtenían calificaciones más bajas. A pesar de que la satisfacción global obtenida en nuestra investigación no correlaciona significativamente con el rendimiento, algunas de las subdimensiones que componen la escala muestran una correlación negativa con el rendimiento, en la línea de los resultados de Hon et al. (2011).

A continuación comentaremos algunas críticas que se deberían considerar de cara a futuras investigaciones sobre el tema. Los resultados hallados, que refutan nuestra hipótesis de partida, pueden estar influidos por diversos factores, entre ellos destacamos:

- Una muestra insuficiente, ya que si hubiésemos reunido un mayor número de participantes, los efectos observados podrían haberse visto modificados.

- El momento económico actual, que puede conllevar a una insatisfacción debida a los recortes en educación. Los alumnos que conforman nuestra muestra pueden encontrarse insatisfechos con ciertas desventajas que están sufriendo un gran número de estudiantes españoles, como pueden ser la disminución del número y la cuantía de becas y ayudas para el estudio, aumento del porcentaje de créditos superados exigidos para poder acceder a dichas ayudas, problemas en la gestión del personal docente (demora de la incorporación de los profesores a su puesto de trabajo una vez comenzado el año escolar).

- El cambio de plan de estudio de Licenciatura a Grado es posible que genere cierta incertidumbre. Algunos ejemplos son la extinción de asignaturas año tras año, viéndose obligados a cursar asignaturas que no son de su interés, el uso de métodos de instrucción y evaluación de los estudios de Grado para alumnos de Licenciatura, problemas con las plazas para realizar las prácticas, etc. 


\section{Referencias bibliográficas}

Caballero, C.C., Abello, R., y Palacio, J. (2007). Relación del burnout y el rendimiento académico con la satisfacción frente a los estudios en estudiantes universitarios. Avances en Psicología Latinoamericana, 25(2), 98-111.

Cabrera, P y Galán, E. (2002). Satisfacción escolar y Rendimiento académico. Revista de Psicodidáctica, 14, 86-98.

Fernández, J. E., Fernández, S., Álvarez, A. y Martínez, P. (2007). Éxito académico y satisfacción de los estudiantes con la enseñanza universitaria. RELIEVE, 13, (2), 203214.

Garbanzo, G.M. (2007). Factores asociados al rendimiento académico en estudiantes universitarios, una reflexión desde la calidad de la educación superior pública. Revista Educación, 31(1), 43-63.

Gento, S. y Vivas, M. (2003). EI SEUE: un instrumento para conocer la satisfacción de los estudiantes universitarios con su educación. Acción Pedagógica, 12(2), 16- 27.

Hon, Raslin y Thean (2011). Student satisfaction and academic performance: a discriminant analysis approach. Interdisciplinary journal of contemporary research in business, 3(2), 1401-1407.

Hussain, N., y Bhamani, S. (2012). Development of the Student University Satisfaction Scale: Reliabiliy and validity. Interdisciplinary Journal of Contemporary Research in Business, 4 (3). 332-342.

Jiménez, A., Terriquez, B., y Robles, F. J. (2011). Evaluación de la satisfacción académica de los estudiantes de la Universidad Autónoma de Nayarit. Revista Fuente, 3(6), 46-56.

Montero, E., Villalobos, J. y Valverde, A. (2007). Factores institucionales, pedagógicos, psicosociales y sociodemográficos asociados al rendimiento académico en la universidad de Costa Rica: un análisis multinivel. RELIEVE, 13(2), 215-234.

Pereira, M. (2011). Nuevas tendencias en la evaluación de la calidad de las universidades: los índices de calidad percibida y satisfacción de los egresados (con modelos de ecuaciones estructurales). Aula Abierta, 39(3), 73-84.

Salanova, M., Martínez, I.M., Bresó, E., Llorens, S., y Grau R. (2005). Bienestar psicológico en estudiantes universitarios: facilitadores y obstaculizadores del desempeño académico. Anales de Psicología, 21(1), 170-180.

Zas, B. (2002). La satisfacción como indicador de excelencia en la calidad de los servicios de salud. Revista Electrónica Psicología Científica. Recuperado de http://psicologiacientifica.com. 